\title{
One-to-one mappings into the plane
}

by

Dix H. Pettey (Salt Lake City, Utah)

1. Introduction. Kenneth Whyburn [16] and L. C. Glaser ([4], [5], and [6]) have given examples which show that for each $n \geqslant 3$ there is a connected $n$-manifold with boundary which can be taken onto $E^{n}$ by a nontopological 1-1 mapping. This contradicts an asserted theorem of $\mathrm{V}$. V. Proizvolov [11].

It is known (see [11] and [15]) that every 1-1 mapping of a connected, locally peripherally compact topological space onto $E^{1}$ is a homeomorphism. (A space is said to be locally peripherally compact if it has a basis $\mathfrak{B}$ of open sets such that every member of $\mathfrak{B}$ has a compact boundary.)

In [4], Glaser showed that if a connected 2 -manifold with boundary $M^{2}$ is a subset of $I^{2}$, with $\operatorname{Int} M^{2}=\operatorname{Int} I^{2}$, and if a 1-1 mapping $f$ of $M^{2}$ onto $E^{2}$ is continuous in $M M^{2}$ at $I^{2}-M^{2}$ (see [4] for a definition of this last condition), then $f$ is a homeomorphism.

The main result of the present paper (Theorem 5.1) is that every 1-1 mapping of a connected 2 -manifold with boundary onto $E^{2}$ is a homeomorphism. An analogous theorem (Theorem 5.2), in which $E^{2}$ is replaced by $S^{2}$, is also shown to be valid. Finally, we prove a generalization of Theorem 5.1; i.e., if each of $M^{2}$ and $Y^{2}$ is a connected 2 -manifold with boundary, Int $Y^{2}$ is an open 2 - cell, and $f$ is a 1-1 mapping of $M^{2}$ onto $Y^{2}$, then $f$ is a homeomorphism (Theorem 5.3). An immediate corollary to this last result is that every 1-1 mapping of a connected 2 -manifold with boundary onto $I^{2}$ is a homeomorphism (Corollary 5.4).

Some related results, pertaining to 1-1 mappings of connected topological spaces onto $E^{2}$, have been obtained by Edwin Duda [2] and R. F. Dickman, Jr. [1].

2. Definitions and notation. Throughout this paper, $E^{n}$ will denote Euclidean $n$-space. The sets $\left\{\left(x_{1}, \ldots, x_{n}\right) \in E^{n} \mid x_{n} \geqslant 0\right\}$ and $\left\{\left(x_{1}, \ldots, x_{n}\right) \epsilon\right.$ $\left.\epsilon E^{n} \mid x_{n} \leqslant 0\right\}$ will be denoted, respectively, by $E_{+}^{n}$ and $\mathbb{E}_{-}^{n}$. The unit $n$-sphere, denoted by $S_{n}$, is defined to be the set of all points $x$ of $E^{n+1}$ such that the distance from $x$ to the origin is 1 . The unit $n$-cube, denoted by $I^{n}$, is defined to be the set $\left\{\left(x_{1}, \ldots, x_{n}\right) \in E^{n} \mid 0 \leqslant x_{i} \leqslant 1\right.$ for $\left.1 \leqslant i \leqslant n\right\}$. 
A set $C$ in a topological space is said to be an $n$-cell if $C$ is homeomorphic to $I^{n}$. A 2 - cell is called a disc. If a set $O$ in a topological space is homeomorphic to $E^{n}$, then $O$ is said to be an open $n$-cell.

A metric space $M$ is said to be an $n$-manifold if for each point $x$ of $M$, some open neighborhood of $x$ is an open $n$-cell. A metric space $M^{\prime}$ is said to be an $n$-manifold with boundary if for each point $x$ of $M L^{\prime}$, either $x$ is in an open set which is an open $n$-cell or $x$ is in an open set which is homeomorphic to $E_{+}^{n}$. If $M I^{\prime}$ is an $n$-manifold with boundary, then the interior of $M^{\prime}$, denoted by Int $M I^{\prime}$, is defined to be the set of all points $x$ of $M^{\prime}$ such that $x$ is in an open set which is an open $n$-cell; the boundary of $M I^{\prime}$, denoted by $\mathrm{Bd} M I^{\prime}$, is defined to be the set $M^{\prime}-\operatorname{Int} M^{\prime}$

Remark. n-manifold and n-manifold with boundary are sometimes defined without the requirement that such spaces be metric. However, by [12], Theorem 1, a locally connected, locally compact topological space is metrizable if it has a 1-1 continuous image in a metric space. Therefore, the theorems of Sections 4 and 5 are still valid as stated even if these less restrictive definitions are used.

A set $L$ in a topological space is said to be a topological line if $L$ is homeomorphic to $E^{1}$. A set $R$ in a topological space is called a topological ray if $R$ is homeomorphic to $E_{+}^{1}$.

If $K$ is a set in a topological space $S$, then $\mathrm{Cl}(K)$ denotes the closure of $K$ in $S$.

A collection of point sets having more than one member is said to be a nondegenerate collection. If $G$ is a collection of point sets, then by the union of $G$ (or $\cup G$ ) we will mean the union of the members of $G$.

The word mapping, as used in this paper, will always refer to a continuous function.

3. Preliminary theorems. The theorems stated in this section will be used in proving the theorems of Sections 4 and 5 . Some of these preliminary theorems are well-known theorems of point set topology or the immediate consequences of well-known theorems, and in these cases we will usually include references but no proofs. In other cases, where the theorems may be less familiar, proofs will be given.

THEOREM 3.1 (BAIRE's THEOREM). Suppose that $X$ is a nonempty, locally compact Hausdorff space and that $₹$ is a countable collection of closed subsets of $X$ such that $X=\bigcup \mathcal{F}$. Then there is a nonempty open set $O$ in $X$ such that $O$ is a subset of some member of $\widetilde{F}$. (See [3], p. 250.)

THEOREM 3.2. Suppose that each of $M_{1}$ and $M_{2}$ is an $n$-manifold and that $f$ is a 1-1 mapping of $M_{1}$ into $M_{2}$. Then $f$ is a homeomorphic embedding. (This theorem follows easily from Brouwer's theorem on invariance of domain; see [7], pp. 95-96.)
THEOREM 3.3. Suppose that $M_{1}$ is an n-manifold, $M_{2}$ is an n-manifold with boundary, and $f$ is a 1-1 mapping of $M_{1}$ into $M I_{2}$. Then $f$ takes $M_{1}$ homeomorphically into Int $M_{2}$.

Proof. Assume that for some point $x$ of $M_{1}, f(x)$ is in Bd $M_{2}$. Choose an open neighborhood $N$ of $f(x)$ such that $N$ is homeomorphic to $E_{+}^{n}$, and let $h$ be a homeomorphism of $N$ onto $E_{+}^{n}$. Then $h f(x) \epsilon E_{+}^{n} \cap E_{-}^{n}$. Let $O$ be an open set in $M_{1}$ such that $x \in O, O$ is an open $n$-cell, and $\mathrm{Cl}(O)$ is a compact subset of $f^{-1}(N)$. Then hf takes $O$ homeomorphically into $E_{+}^{n}$. Since $O$ is an open $n$-cell, Brouwer's theorem on invariance of domain tells us that $h f(O)$ is open in $E^{n}$. Because $h f(O)$ is a subset of $E_{+}^{n}$, this means that $h f(O)$ does not intersect $E_{+}^{n} \cap E_{-}^{n}$. But $f(x) \in f(O)$ and $h f(x) \in E_{+}^{n} \cap E_{-}^{n}$. This contradiction implies that $f\left(M_{1}\right)$ is a subset of Int $M_{2}$.

Since Int $M_{2}$ is an $n$-manifold, Theorem 3.2 tells us that $f$ takes $M_{1}$ homeomorphically into Int $M_{2}$.

THEOREM 3.4. Suppose that each of $M_{1}$ and $M_{2}$ is an n-manifold with boundary and that $f$ is a 1-1 mapping of $M_{1}$ into $M_{2}$ such that $f\left(\mathrm{Bd} M_{1}\right) \mathrm{C}$ B.d $M_{2}$. Then $f$ is a homeomorphic embedding.

Proof. Let $x$ be a point of $\mathrm{Bd} M_{1}$ and let $U$ be an open set containing $x$. Choose an open set $O$ in $M_{2}$ such that $f(x) \in O$ and such that $O$ is homeomorphic to $E_{+}^{n}$. Let $\varphi$ denote a homeomorphism of $O$ onto $E_{+}^{n}$. Let $N$ denote an open neighborhood of $x$ such that $N$ is homeomorphic to $E_{+}^{n}$ and such that $\mathrm{Cl}(N)$ is a compact subset of $U \cap f^{-1}(O)$. Let $\theta$ be a homeomorphism of $E_{+}^{n}$. onto $N$. Then $\varphi f \theta$ is a homeomorphism of $E_{+}^{n}$ into itself such that $\varphi f \theta\left(E_{+}^{n} \cap E_{-}^{n}\right) \subset E_{+}^{n} \cap E_{-}^{n}$. Let $h$ be a homeomorphism of $E_{-}^{n}$ into itself such that $\varphi f \theta\left|E_{+}^{n} \cap E_{-}^{n}=h\right| E_{+}^{n} \cap E_{-}^{n}$. Define the function $H$ of $E^{n}$ into itself as follows:

$$
H(x)=\left\{\begin{array}{lll}
h(x) & \text { if } & x \in E_{-}^{n}, \\
\varphi f \theta(x) & \text { if } & x \in E_{+}^{n}
\end{array}\right.
$$

Clearly, $H$ is a homeomorphism of $E^{n}$ into itself. By Brouwer's theorem on invariance of domain, then, $H\left(E^{n}\right)$ is open in $E^{n}$. Consequently, $H\left(E_{+}^{n}\right)$ is open in $E_{+}^{n}$, i.e., $\varphi f(N)$ is open in $E_{+}^{n}$. This implies that $f(N)$ is open in $M_{2}$. Hence, for every point $x$ of $\mathrm{Bd} M_{1}$ and every open set $U$ containing $x$, there is an open neighborhood $N$ of $x$ such that $N \subset U$ and $f(N)$ is open in $M_{2}$.

By Theorem 3.3, Int $M_{1}$ is taken homeomorphically by $f$ into Int $M_{2}$. Thus, for every point $x$ of Int $M_{1}$ and every open set $U$ containing $x$, there is an open neighborhood $N$ of $x$ such that $N \subset U$ and $f(N)$ is open in $M_{2}$. We conclude, then, that $f$ is an open mapping, and this implies that $f$ is a homeomorphic embedding. 
THEOREM 3.5. An n-manifold with boundary is connected if and only if its interior is connected. (This theorem is an immediate consequence of the definition of $n$-manifold with boundary.)

THEOREM 3.6. If $M^{1}$ is a nonempty 1-manifold, then every component of $M^{1}$ is either a simple closed curve or a topological line. (See [14], p. 194.)

In [9], Janiszewski showed that if each of $H_{1}$ and $H_{2}$ is a closed, connected, bounded set in $E^{2}$, and if $H_{1} \cap H_{2}$ is disconnected, then $H_{1} \cup H_{2}$ separates $E^{2}$. Each of the following two theorems is easily obtained from Janiszewski's result by use of the method of inversion. (See [10], p. 36, for an illustration of this method.)

THEOREM 3.7. Suppose that each of $H_{1}$ and $H_{2}$ is a closed, connected subset of $E^{2}$ and that $H_{1} \cap H_{2}$ is a disconnected, bounded set. Then $H_{1} \cup H_{2}$ separates $E^{2}$.

THEOREM 3.8. Suppose that each of $H_{1}$ and $H_{2}$ is a closed, connected, unbounded subset of $E^{2}$ and that $H_{1} \cap H_{2}$ is nonempty and has a bounded component. Then $H_{1} \cup H_{2}$ separates $E^{2}$.

4. Mappings onto separating sets in $E^{2}$. The main results of this section are Theorems 4.7 and 4.8 , each of which is important in proving the main theorems of Section 5. Since Theorem 4.8 follows quite easily from Theorem 4.7, most of the material in this section is directed toward the proving of Theorem 4.7. The first six theorems of the section are included primarily as lemmas to be used for this purpose.

THEOREM 4.1. Suppose that the nonempty set $H$ in $E^{2}$ is the union of a countable collection $G$ of arcs and that $\mathrm{Cl}(H)-H$ is the union of countably many closed sets. Then there is an open set $O$ in $E^{2}$ such that $O \cap \mathrm{Cl}(H)$ is a topological line and a subset of some member of $G$.

Proof. Let $\mathfrak{F}$ be a countable collection of closed sets such that $\cup \widetilde{F}=\mathrm{Cl}(H)$ and such that each member of $\mathfrak{F}$ which intersects $H$ is a member of $G$. Because $\mathrm{Cl}(H)$ is a closed subset of $E^{2}, \mathrm{Cl}(H)$ is locally compact. Hence, by Theorem 3.1, there is a nonempty open set $O^{\prime}$ in $E^{2}$ such that $O^{\prime} \cap \mathrm{Ol}(H)$ is a subset of some member $F$ of $\mathfrak{F}$. Since every point of $\mathrm{Ol}(H)-H$ is a limit point of $\bigcup G$, it follows that $F \in G$. Therefore, since each member of $G$ is an are, there is an open subset $O$ of $O^{\prime}$ such that $O \cap F^{\prime}(=O \cap \mathrm{Cl}(H))$ is a topological line.

THEOREM 4.2. Suppose that the nonempty set $H$ in $E^{2}$ is the union of countably many ares and that $\mathrm{Cl}(H)-H$ is the union of countably many closed sets. Then $\mathrm{CI}(\boldsymbol{H})$ is nowhere dense in $E^{2}$.

Proof. Let $\mathfrak{F}$ be a countable collection of closed sets such that $\cup \mathfrak{F}=\mathrm{Ol}(H)$ and such that every member of $\widetilde{F}$ which intersects $H$ is an are in $H$. Since every member of $\mathfrak{F}$ is either an are or a subset of $\mathrm{Cl}(H)-H$, it follows that no dise in $E^{2}$ is a subset of a member of $\mathfrak{F}$; i.e., each member of $\mathfrak{F}$ is nowhere dense in $E^{2}$. Let $D$ be a dise in $E^{2}$. Then, for each $F \in \mathfrak{F}, F \cap D$ is nowhere dense in $D$. Therefore, since $D$ is compact, $D \neq \bigcup_{F \in \mathcal{F}}(F \cap D)$; i.e., $D$ is not a subset of $\bigcup \mathcal{F}$. This implies that $\bigcup \widetilde{F}(=\mathrm{Cl}(H))$ is nowhere dense in $B^{2}$.

THEOREM 4.3. Suppose that the nonempty set $H$ in $E^{2}$ is the union of countably many arcs and that $\mathrm{Cl}(H)-H$ is the union of countably many closed sets. Suppose, furthermore, that some subset $K$ of $\mathrm{Cl}(H)$ separates $E^{2}$. Then $\mathrm{Cl}(H)$ separates $E^{2}$.

Proof. Assume that $\mathrm{Cl}(H)$ does not separate $E^{2}$. Let $B$ and $C$ denote two mutually separated nonempty sets such that $E^{2}-K=B \cup C$. Let $O$ denote an open set which intersects $B$ but not $C$, and let $O^{\prime}$ denote an open set which intersects $C$ but not $B$. It follows from Theorem 4.2 that there is a point $x$ of $E^{2}-\mathrm{Cl}(H)$ in $O$ and a point $x^{\prime}$ of $E^{2}-\mathrm{Cl}(H)$ in $O^{\prime}$. Since $\mathrm{Cl}(H)$ does not separate $E^{2}$, there is an are $A$ from $x$ to $x^{\prime}$ in $E^{2}-\mathrm{Cl}(H)$. Then $A$ is a connected subset of $B \cup C$. But, since $x \in[O \cap(B \cup C)] \subset B$ and $x^{\prime} \in\left[O^{\prime} \cap(B \cup C)\right] C C$, this is impossible. Hence, the assumption that $\mathrm{Cl}(H)$ does not separate $E^{2}$ has led to a contradiction.

THEOREM 4.4. Suppose that the topological space $S$ is the union of a finite, nondegenerate collection $\left\{R_{1}, R_{2}, \ldots, R_{n}\right\}$ of disjoint topological rays and that for each $i(i=1,2, \ldots, n) p_{i}$ is the end-point of $R_{i}$. Suppose, furthermore, that $f$ is a 1-1 mapping of $S$ into $E^{2}$ such that

(1) for $i=1,2, \ldots, n-1, f\left(p_{i+1}\right)$ is a limit point of $f\left(R_{i}\right)$,

(2) $f\left(p_{1}\right)$ is a limit point of $f\left(R_{n}\right)$,

(3) $\mathrm{Cl}[f(S)]-f(S)$ is the union of countably many closed sets.

Then $\mathrm{Cl}[f(S)]$ separates $E^{2}$.

Proof. For each $i(i=1,2, \ldots, n) R_{i}$ is the union of countably many arcs. Therefore, since $f$ is $1-1$ and continuous, $f(S)$ is the union of a countable collection $G$ of ares, each of which is the homeomorphic image (under $f$ ) of an are in some $R_{i}$. It follows, then, from Theorem 4.1 that there is an open set $O$ in $E^{2}$ such that $O \cap \mathrm{Cl}[f(S)]$ is a topological line and a subset of some member of $G$. Let $H^{\prime}$ be an are in $O \cap C \mathrm{I}[f(S)]$, and let $H^{\prime \prime}$ denote the closure of $f(S)-H^{\prime}$. Clearly, $H^{\prime \prime}$ is connected. Since $H^{\prime} \cap H^{\prime \prime}$ consists of the two end-points of $H^{\prime}$, Theorem 3.7 implies that $H^{\prime} \cup H^{\prime \prime}$ separates $E^{2}$, i.e., $\mathrm{Cl}[f(S)]$ separates $E^{2}$.

THEOREM 4.5. Suppose that the topological space $S$ is the union of a finite, nondegenerate collection $\left\{L_{1}, L_{2}, \ldots, L_{n}\right\}$ of disjoint topological lines and that $f$ is a 1-1 mapping of $S$ into $E^{2}$ such that

(1) for $i=1,2, \ldots, n-1, f\left(L_{i+1}\right)$ contains a limit point $q_{i+1}$ of $f\left(L_{i}\right)$,

(2) $f\left(L_{1}\right)$ contains a limit point $q_{1}$ of $f\left(L_{n}\right)$,

(3) $\mathrm{Cl}[f(S)]-f(S)$ is the union of countably many closed sets.

Then $\mathrm{OI}[f(S)]$ separates $E^{2}$. 
Proof. For $i=1,2, \ldots, n-1$, there is a topological ray $R_{i}$ in $L_{i}$ such that $f^{-1}\left(q_{i}\right)$ is the end-point of $R_{i}$ and $q_{i+1}$ is a limit point of $f\left(R_{i}\right)$. Similarly, there is a topological ray $R_{n}$ in $L_{n}$ such that $f^{-1}\left(q_{n}\right)$ is the endpoint of $R_{n}$ and $q_{1}$ is a limit point of $f\left(R_{n}\right)$. Let $S^{\prime}=\bigcup_{i=1}^{n} R_{i}$. Now for each $i(i=1,2, \ldots, n), L_{i}-R_{i}$ is the union of countably many arcs. Therefore, since $f$ is $1-1$ and continuous, $f\left(S-S^{\prime}\right)\left(=f(S)-f\left(S^{\prime}\right)\right)$ is the union of countably many arcs. This, together with Condition (3) of the hypothesis, implies that $\mathrm{Cl}\left[f\left(S^{\prime}\right)\right]-f\left(S^{\prime}\right)$ is the union of countably many closed sets and, consequently, that $\mathrm{Cl}\left[f\left(S^{\prime}\right)\right]-f\left(S^{\prime}\right)$ is the union of countably many closed sets. Therefore, by Theorem 4.4, $\mathrm{Cl}\left[\left(S^{\prime}\right)\right]$ separates $E^{2}$. By Theorem 4.3, then, $\mathrm{Cl}[f(S)]$ separates $E^{2}$.

THEOREM 4.6. Suppose that $L$ is a topological line and that $f$ is a 1-1 mapping of $L$ into $E^{2}$ such that $\mathrm{Cl}[f(L)]-f(L)$ is the union of countably many closed sets. Then either $f$ is a homeomorphism or $\mathrm{Cl}[f(L)]$ separates $E^{2}$.

Proof. Assume that $f$ is not a homeomorphism. Then there is a point $p_{1}$ of $L$ and a sequence $\left\langle x_{i}\right\rangle$ of points of $L$ such that no subsequence of $\left\langle x_{i}\right\rangle$ converges in $L$ but $\left\langle f\left(x_{i}\right)\right\rangle$ converges to $f\left(p_{1}\right)$ in $E^{2}$. Let $R$ be a topological ray in $L$ such that $p_{1}$ is an end-point of $R$ and $R$ contains infinitely many points of $\left\langle x_{i}\right\rangle$. Let $p_{2}$ be a point of $R-p_{1}$, and let $R_{1}$ and $R_{2}$ be disjoint topological rays such that $R=R_{1} \cup R_{2}, p_{1}$ is the endpoint of $R_{1}$, and $p_{2}$ is the end-point of $R_{2}$. Then $f\left(p_{2}\right)$ is a limit point of $f\left(R_{1}\right)$, and $f\left(p_{1}\right)$ is a limit point of $f\left(R_{2}\right)$. Since $L-R$ is the union of countably many ares, $\mathrm{C}[f(L)]-f(R)$ is the union of countably many closed sets, and, consequently, $\mathrm{Cl}[f(R)]-f(R)$ is the union of countably many closed sets. By Theorem 4.4, then, $\mathrm{Cl}[f(R)]$ separates $E^{2}$. Therefore, by Theorem $4.3, \mathrm{Cl}[f(L)]$ separates $E^{2}$.

Remark. Without the requirement that $\mathrm{Cl}[f(L)]-f(L)$ be the union of countably many closed sets, Theorem 4.6 is no longer valid. For example, we can easily construct a nontopological 1-1 mapping of $E^{1}$ into $E^{2}$ such that the closure of the image of $E^{1}$ is the indecomposable, nonseparating plane continuum illustrated in [8], Figure 7. Another example is obtained by constructing a 1-1 nontopological mapping $f$ of $E^{1}$ into $E^{2}$ such that $f\left(E^{1}\right) \subset \operatorname{Int} I^{2}$ and such that $f\left(E^{1}\right)$ contains a countable dense subset of $I^{2}$; we then have $\operatorname{Cl}\left[f\left(E^{1}\right)\right]=I^{2}$.

THEOREM 4.7. If $M^{1}$ is a nonempty separable 1 -manifold, $f$ is a 1-1 mapping of $M^{1}$ into $E^{2}$, and $f\left(M^{1}\right)$ is closed in $E^{2}$, then $f\left(M^{1}\right)$ separates $E^{2}$.

Proof. Assume that $f\left(M^{1}\right)$ does not separate $E^{2}$.

Since $M^{1}$ is a separable 1-manifold (and is therefore the union of countably many arcs), and since $f$ is $1-1$ and continuous, it follows that $f\left(M^{1}\right)$ is the union of countably many arcs. Theorem 4.3, then, implies that no subset of $f\left(M^{1}\right)$ separates $E^{2}$. From this we conclude that $f\left(M^{1}\right)$ contains no simple closed curve. Therefore, if follows from Theorem 3.6 that every component of $M K^{1}$ is a topological line. Let $\pi$ denote the collection of all components of $M^{1}$, and let $G$ denote the collection $\{L \mid L=f(K)$ for some $K \in \pi\}$.

If $\pi^{\prime}$ is a subcollection of $\pi$, then $M^{1}-\bigcup \pi^{\prime}$ is the union of countably many ares, and this implies that $f\left(M^{1}\right)-f\left(\cup \pi^{\prime}\right)$ is the union of countably many arcs. Since $f\left(M K^{1}\right)$ is closed in $E^{2}$, we conclude that for each subcollection $\varkappa^{\prime}$ of $\pi, \mathrm{Cl}\left[f\left(\bigcup \varkappa^{\prime}\right)\right]-f\left(\bigcup \varkappa^{\prime}\right)$ is the union of countably many closed sets. For each $K \in \pi$, then, $\mathrm{Cl}[f(K)]-f(K)$ is the union of countably many closed sets. Therefore, since no subset of $f\left(M^{1}\right)$ separates $E^{2}$, it follows from Theorem 4.6 that for each $K \in \pi, f \mid K$ is a homeomorphism, i.e., each element of $G$ is the homeomorphic image, under $f$, of some element of $\pi$.

Now let $L_{1}, L_{2}, \ldots$ denote the elements of $G$. For each $i(i=1,2, \ldots)$, let $h_{i}$ be a homeomorphism of $E^{1}$ onto $L_{i}$, and define the following sets and collections of sets as indicated:

$$
\begin{aligned}
L_{i}^{+} & =h_{i}\left(E_{+}^{1}\right) \quad \text { and } \quad L_{i}^{-}=h_{i}\left(E_{-}^{1}\right) ; \\
\mathfrak{J}_{i}^{+}(1) & =\left\{\left.L \in G\right|_{,} L \neq L_{i}, L \frown \mathrm{Cl}\left(L_{i}^{+}\right) \neq \emptyset\right\}
\end{aligned}
$$

and

$$
\widetilde{J}_{i}^{\bar{i}}(1)=\left\{L \in G \mid L \neq L_{i}, L \cap \mathrm{CL}\left(L_{i}^{-}\right) \neq \emptyset\right\}
$$

for $n=1,2, \ldots$

$$
\mathfrak{J}_{i}^{+}(n+1)=\left\{L \in G \mid L \cap \mathrm{Cl}\left(L^{\prime}\right) \neq \emptyset \text { for some } L^{\prime} \in \mathfrak{S}_{i}^{+}(n)\right\}
$$

and

$$
\begin{aligned}
& \widetilde{J}_{i}^{-}(n+1)=\left\{L \in G \mid L \cap \mathrm{Cl}\left(L^{\prime}\right) \neq \emptyset \text { for some } L^{\prime} \in \mathfrak{S}_{i}(n)\right\} ; \\
& \mathfrak{\Im}_{i}^{+}=\bigcup_{n=1}^{\infty} \mathfrak{\Im}_{i}^{+}(n) \quad \text { and } \quad \mathfrak{\Im}_{i}=\bigcup_{n=1}^{\infty} \mathfrak{I}_{i}^{-}(n) \\
& \widetilde{J}_{i}=\widetilde{\Im}_{i}^{+} \cup \mathfrak{\Im}_{i}^{-} \cup\left\{L_{i}\right\} ; \\
& V_{i}^{+}=\mathrm{Cl}\left(\cup \mathfrak{S}_{i}^{+}\right), \quad V_{i}^{-}=\mathrm{Cl}\left(\cup \widetilde{S}_{i}^{-}\right), \quad \text { and } \quad V_{i}=\mathrm{Cl}\left(\bigcup \mathfrak{I}_{i}\right) .
\end{aligned}
$$

It is clear that whenever $L_{j} \in \widetilde{J}_{i}$ we will have $\mathfrak{J}_{j} \subset \mathfrak{J}_{i}$ and $V_{j} \subset \nabla_{i}$. It follows from Theorem 4.5 that there does not exist a finite, nondegenerate collection $\left\{K_{1}, K_{2}, \ldots, K_{m}\right\}$ of elements of $\pi$ such that $f\left(K_{1}\right)$ contains a limit point of $f\left(K_{m}\right)$ and such that for each $i(i=1,2, \ldots, m-1)$ $f\left(K_{i+1}\right)$ contains a limit point of $f\left(K_{i}\right)$. Using this fact we are able to conclude the following:

(1) if $L_{j} \in \mathfrak{J}_{i}$ and $i \neq j$, then $L_{i} \notin \mathfrak{J}_{j}$; and

(2) for each positive integer $i, L_{i} \notin \mathfrak{J}_{i}^{+} \cup \mathfrak{J}_{i}^{-}$. 
Now for each positive integer $i, \Im_{i}$ is a subcollection of $G$, and therefore,

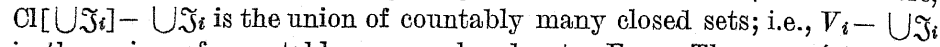
is the union of countably many closed sets. From Theorem.4.1, then, it follows that there is an open set $O$ in $E^{2}$ such that $O \cap V_{i}$ is a topological line and a subset of some member of $\mathfrak{I}_{i}$.

Since $V_{j} \subset V_{i}$ whenever $L_{j} \epsilon \mathfrak{J}_{i}$, we can choose a positive integer $k$ such that for some open set $O$ in $E^{2}, O \cap V_{k}$ is a topological line and a subset of $L_{k}$.

Case 1: $V_{k}^{+} \cup L_{k}^{+}$is bounded. Then $\mathfrak{J}_{k}^{+}$is non-empty, and, for each $j$ such that $L_{j} \in \mathfrak{I}_{k}^{+}$, neither $\widetilde{J}_{j}^{+}$nor $\mathfrak{J}_{j}^{-}$is empty. There exists an integer $m$ such that $L_{m} \in \mathfrak{J}_{k}^{+}$and $L_{1} \notin \mathfrak{I}_{m}$. (If $L_{1} \notin \mathfrak{J}_{k}^{+}$then $m$ can be any integer such that $L_{m} \in \mathfrak{I}_{k}^{+}$; if $L_{1} \in \mathfrak{J}_{k}^{+}$choose $m$ such that $L_{m} \in \mathfrak{I}_{1}^{+}$.) There exists an integer $r(1)$ such that $L_{r(1)} \in \widetilde{J}_{m}$ and such that for some open set $O^{\prime}$ in $E^{2}$, $O^{\prime} \cap \nabla_{r(1)}$ is a topological line and a subset of $L_{r(1)}$. One of the sets $V_{r(1)}^{+}, V_{r^{(1)}}^{-}$does not intersect $L_{1}$. (For if $L_{1}$ intersected each of $V_{r^{(1)}}^{+}$ and $V_{r(1)}^{-}$, there would then be an arc $A$ in $L_{1}$ intersecting each of $V_{r(1)}^{+1}$ and $V_{r^{(1)}}$. Then Theorem 3.7, along with the fact that $O^{\prime} \cap V_{r(1)}$ is a topological line in $L_{r(1)}$, would imply that $A \cup V_{r(1)}$ separates $E^{2}$.) Let $R_{1}$ denote a member of $\left\{\nabla_{r_{(1)}}^{+}, \nabla_{r_{(1)}}^{-}\right\}$which does not intersect $L_{1}$. Similarly, we can find an integer $r(2)$ such that $V_{r(2)} \subset R_{1}$ and such that one of the sets $\nabla_{r(2)}^{+}, \nabla_{r(2)}^{-}$does not intersect $L_{2}$. Let $R_{2}$ denote a member of $\left\{V_{r(2)}^{+}, V_{r(2)}^{-}\right\}$which does not intersect $L_{2}$. Continuing in this manner, we define a sequence $\left\langle R_{i}\right\rangle$ of nonempty, compact sets such that for each $i, R_{i+1} \subset R_{i}$ and $R_{i} \cap L_{i}=\emptyset$. Then $\bigcap_{j=1}^{\infty} R_{j} \neq \emptyset$. But clearly $\bigcap_{j=1}^{\infty} R_{j}$ can intersect no $L_{i}$. Since $f\left(M^{1}\right)$ is the union of the $L_{i}$ 's and since each $R_{j}$ is a subset of $f\left(M^{1}\right)$, this gives us a contradiction.

Case 2: $\nabla_{k}^{-} \cup L_{k}^{-}$is bounded. This case is analogous to Case 1 and, therefore, also leads to a contradiction.

Case 3: Each of $V_{k}^{+} \cup L_{k}^{+}$and $V_{k}^{-} \cup L_{k}^{-}$is unbounded. Then, from Theorem 3.8 and the fact that $O \cap V_{k}$ is a topological line in $L_{k}$, it follows that $V_{k}$ separates $E^{2}$. This, again, is a contradiction.

Since each of the three possible cases results in a contradiction, we conclude that our original assumption is false; i.e., $f\left(M^{1}\right)$ separates $E^{2}$.

THEOREM 4.8. If $M^{1}$ is a nonempty separable 1-manifold, $f$ is a 1-1 mapping of $M^{1}$ into $S^{2}$, and $f\left(M^{1}\right)$ is closed in $S^{2}$, then $f\left(M^{1}\right)$ separates $S^{2}$.

Proof. Assume that $f\left(M^{1}\right)$ does not separate $S^{2}$. Since $f\left(M^{1}\right)$ is the union of countably many arcs, it follows from Baire's Theorem that $f\left(M^{1}\right) \neq S^{2}$. Thus, $S^{2}-f\left(M^{1}\right)$ is a nonempty, connected, open subset of $S^{2}$. Choose $p \in\left[S^{2}-f\left(M^{1}\right)\right]$. Since $S^{2}-f\left(M^{1}\right)$ is a connected open set in $S^{2}, p$ does not separate $S^{2}-f\left(M^{1}\right)$. Therefore, $S^{2}-\left[p \cup f\left(M^{1}\right)\right]$ is connected. But, since $S^{2}-p$ is homeomorphic to $E_{2}$, Theorem 4.7 implies that $f\left(M^{1}\right)$ separates $S^{2}-p$, i.e., that $S^{2}-\left[p \cup f\left(M^{1}\right)\right]$ is not connected. Hence, we have a contradiction.

5. 1-1 mappings of 2-manifolds with boundary. In this section we will consider a 1-1 mapping $f$ of a connected 2-manifold with boundary onto a space $Y$. Theorem 5.1 says that if $Y$ is $E^{2}$, then $f$ must be a homeomorphism. Theorem 5.2 asserts that the same is true if $Y$ is $S^{2}$. Theorem 5.3 is a generalization of Theorem 5.1 in which we require only that $Y$ be a 2 -manifold with boundary such that Int $Y$ is an open 2-cell.

THEOREM 5.1. Suppose that $M^{2}$ is a connected 2-manifold with boundary and that $f$ is a 1-1 mapping of $M^{2}$ onto $E^{2}$. Then $f$ is a homeomorphism.

Proof. Assume that $M^{2}$ has a nonempty boundary. It follows from [13], Corollary, p. 111, that $M^{2}$ is a separable metric space. Hence, Bd $M^{2}$ is a separable 1-manifold. For each point $x$ of Int $M^{2}$, there is an open set $O$ in Int $M^{2}$ such that $x \in O$ and $f(O)$ is open in $E^{2}$. Therefore, $f\left(\right.$ Int $\left.M M^{2}\right)$ is open in $E^{2}$, and $f\left(\mathrm{Bd} M^{2}\right)$ is closed in $E^{2}$. By Theorem 4.7, then, $f\left(\right.$ Bd $\left.M K^{2}\right)$ separates $E^{2}$, i.e., $f\left(\right.$ Int $\left.M^{2}\right)$ is not connected. But by Theorem 3.5, Int $M^{2}$ is connected, and, since $f$ is continuous, this means that $f\left(\right.$ Int $M I^{2}$ ) must also be connected. This contradiction implies that our assumption is false; i.e., $\mathrm{Bd} M^{2}=\varnothing$. By Theorem 3.2, then, $f$ is a homeomorphism.

THEOREM 5.2. Suppose that $M^{2}$ is a connected 2-manifold with boundary and that $f$ is a 1-1 mapping of $M^{2}$ onto $S^{2}$. Then $f$ is a homeomorphism.

The proof of Theorem 5.2 is analogous to that of Theorem 5.1. Theorem 4.8 is used instead of Theorem 4.7 .

THEOREM 5.3. Suppose that each of $M^{2}$ and $X^{2}$ is a 2-manifold with boundary, that $M^{2}$ is connected, and that $\operatorname{Int} Y^{2}$ is an open 2-cell. Suppose, furthermore, that $f$ is a 1-1 mapping of $M^{2}$ onto $Y^{2}$. Then $f$ is a homeomorphism.

Proof. Since $f^{-1}\left(\operatorname{Int} Y^{2}\right)$ is an open subset of $M^{2}, f^{-1}\left(\operatorname{Int} Y^{2}\right)$ is a 2-manifold with boundary. By Theorem 3.5, Int $M^{2}$ is connected, and by Theorem $3.3, f\left(\operatorname{Int} M^{2}\right) \subset \operatorname{Int} Y^{2}$. Therefore, Int $M^{2} \subset f^{-1}\left(\operatorname{Int} Y^{2}\right)$, and from this it follows that $f^{-1}\left(\operatorname{Int} Y^{2}\right)$ is connected. By Theorem 5.1, then, $f^{-1}\left(\operatorname{Int} Y^{2}\right)$ is taken homeomorphically onto Int $Y^{2}$. This means that $f^{-1}\left(\operatorname{Int} Y^{2}\right)$ is an open 2-cell and, consequently, that Bd $M^{2}$ does not intersect $f^{-1}\left(\operatorname{Int} Y^{2}\right)$. It now follows from Theorem 3.4 that $f$ is a homeomorphism.

COROLUART 5.4. Suppose that $M^{2}$ is a connected 2-manifold with boundary and that $f$ is a 1-1 mapping of $M^{2}$ onto $I^{2}$. Then $f$ is a homeomorphism. 
[1] R. F. Dickman, Jr., Compactness of mappings on products of locally connected generalized continua, Proc. Amer. Math. Soc. 18 (1967), pp. 1093-1094.

[2] Edwin Duda, A theorem on one-to-one mappings, Pacific J. Math. 19 (1966) pp. 253-257.

[3] James Dugundji, Topology, Boston, 1966.

[4] L. C. Glaser, 1-1 continuous mappings onto $E^{n}$, Amer. J. Math. 88 (1966), pp. $237-243$.

[5] - Dimension lowering monotone non-compact mappings of $E^{n}$, Fund. Math. 58 (1966) pp. 177-181.

[6] - Bing's house with two rooms from a $1-1$ continuous map onto $E^{3}$, Amer. Math. - Monthly 74 (1967), pp. 156-160.

[7] Witold Hurewicz and Henry Wallman, Dimension theory, 2nd ed., Princeton University Mathematical Series 4, Princeton, N. J., 1948.

[8] S. Janiszewski, Sur les continus irréductibles entre deux points, J. Ecole Polytech. (2) 16 (1912), pp. 79-170.

[9] - Sur les coupures du plan faites par des continus, Prace Mat.-Fiz. 26 (1913). pp. 11-63.

[10] B. Knaster and C. Kuratowski, Sur les continus nonbornés, Fund. Math 5 (1924), pp. 23-58.

[11] V. V. Proizvolov, Contractions into Euclidean spaces, Soviet Math. Dokl. 4 (1963), pp. 1194-1195, Amer. Math. Soc. translation from Dokl. Akad. Nauk SSSR 151 (1963), pp. 1286-1287.

[12] - One-to-one mappings onto metric spaces, Soviet Math. Dokl. 5 (1964), pp. 13211322, Amer. Math. Soc. translation from Dokl. Akad. Nauk. SSSR I58 (1964) pp. 788-789.

[13] W. Sierpiński, Sur les espaces métriques localement séparables, Fund. Math. 21 (1933), pp. 107-113.

[14] G. T. Whyburn, Analytic topology, 2nd ed., Amer. Math. Soc. Colloquium, Publications 28, Providence, R. I., 1963. [15] - On compactness of mappings, Proc. Nat. Acad. Sci. U.S.A. 52 (1964),
pp. 1426-1431. [16] Kenneth Whyburn, A nontopological 1 -1 mapping onto $E^{3}$, Bull. Amer. Math.
Soc. 71 (1965), pp. 533-537.

UNIVERSITY OF UTAH

Regu par la Rédaction le 22. 8. 1968

\section{A CBV image of a universal null set need not be a universal null set}

by

In [3], the author very naively lists six properties which a subset $E$ of the interval $I=[0,1]$ might possess. None of these properties is less stringent than its successor, and the sixth property is that of being a universal null set:

(6) If $\mu$ is a non-negative non-atomic finite Baire measure on $I$, then $\mu(E)=0$.

Several remarks about whether a listed property is equivalent to or stricter than its successor are given in [3], which was written while the author sought to answer the following question.

If $E$ is a subset of I with property (6) and $h$ is a continuous function of bounded variation on $I$ (CBV); then must $h(E)$ have property $(6)$ ?

Unfortunately, the author was ignorant of a vast amount of pertinent information that was available. For example, A. S. Besicovitch ([2]) information that was available. For example, A. S. Besicoviteh ( becoming aware of Besicovitch's result, the author found another proof of becoming a warion to settle the original question or to show that property (5) does not imply property (4). However, W. Sierpiński that property (5) does a continuous image of a universal null set $([4]$, p. 57) had shown that a continuous image of a universal null se need not be a universal null set, from which it follows that (5) does not imply (4).

The purpose of this note is to answer our question in the negative by modifying Sierpiński's construction so that a theorem of N. Bary can be applied.

Let $P$ be the Cantor set in $I$ of measure $1 / 2$ which is obtained by taking out middle quarters. Let $I_{i j}, j \leqslant 2^{i}$, denote the $i$ th stage intervals in the canonical representation of $P$ as an intersection of finite unions. of intervals, and let $O_{i j}, j \leqslant 2^{i-1}$, denote the corresponding segments which are removed at the $i$ th stage. 